\title{
Laparoscopic versus Open Partial Nephrectomy: Comparison of Overall and Subgroup Outcomes
}

\author{
MOHAMED ABDELHAFEZ ${ }^{1,2}$, AMEND BASTIAN ${ }^{1}$, STEFFEN RAUSCH ${ }^{1}$, \\ ARNULF STENZL $^{1}$, JENS BEDKE $^{1}$ and STEPHAN KRUCK ${ }^{1}$ \\ ${ }^{1}$ Department of Urology, Eberhard Karls University, Tuebingen, Germany; \\ ${ }^{2}$ Department of Urology, Assiut University, Assiut, Egypt
}

\begin{abstract}
Background: At experienced centers, laparoscopic partial nephrectomy (LPN) can achieve similar results to those of open surgery $(O P N)$. However, the role of $L P N$ for complex tumors and imperative indications is under debate. Patients and Methods: A total of 356 cases (186 $L P N$ and $170 O P N)$ between 2005-2012 were reviewed. Clinical, surgical, pathological and radiological data, including PADUA classification were analyzed. Results: In overall analysis, OPN was associated with higher tumor complexity ( $p \leq 0.03)$. Subgroup analysis of PADUA $>8$ tumors $(n=85)$ showed no significant difference between $L P N$ and OPN. In patients with unfavorable treatment characteristics (imperative indication/multifocal tumors, $n=71) L P N$ was beneficial. In this subgroup, LPN led to better perioperative $(p \leq 0.02)$ and postoperative $(p \leq 0.04)$ outcome. Conclusion: Use of LPN is associated with favorable tumor characteristics. Although no advantage was shown for LPN for tumors with higher complexity $(P A D U A>8)$, this large series confirmed the superiority of $L P N$ for imperative indication or multifocal tumors.
\end{abstract}

The European Association of Urology guidelines for renal cell carcinoma (RCC) recommended nephron-sparing surgery (NSS) as the standard procedure for removing small cT1a-stage renal tumors (1). This indication has been extended to include all renal tumors whenever NSS is possible (2) as recent reports indicate that NSS provides an equivalent oncological efficacy and superior renal functional outcomes in advanced stages (T2 and T3a) when compared with radical nephrectomy (3-5). The open approach is the

Correspondence to: Jens Bedke, Eberhard Karls University Tuebingen, Department of Urology, Hoppe-Seyler-Strasse 3, 72076 Tuebingen, Germany. Tel: +49 70712986613, Fax: +49 7071294638, e-mail: jens.bedke@med.uni-tuebingen.de

Key Words: Laparoscopy, partial nephrectomy, renal cell carcinoma, renal score, perioperative outcomes. standard technique for NSS. However, with advances in video technology, laparoscopic instrumentation design, and availability of hemosealant substances, the laparoscopic approach has gained popularity in NSS. Currently there is no contraindication for laparoscopic NSS other than lack of surgeon experience (6-8). Therefore, the superiority of laparoscopic NSS regarding short- and long-term outcomes is still questioned for complex tumors and imperative indications. While earlier reports showed that laparoscopic NSS had more complications and longer operative time than open NSS, recent reports found no difference between the two approaches $(9,10)$. Moreover, some reports indicate that laparoscopic NSS was associated with fewer complications, shorter ischemia time and shorter hospital stay in comparison to open surgery $(7,11-14)$.

The current study analyzed perioperative functional outcomes, primarily defined as the intraoperative and postoperative complication rate, at a large single-center comparison between laparoscopic and open NSS. The reported results will help assess risks in relation to potential benefits for patients with increased tumoral complexity or imperative indication.

\section{Patients and Methods}

Between January 2005 and June 2012, all consecutive patients $(n=361)$ who underwent OPN or LPN at the Department of Urology, University Hospital Tuebingen, Germany, were included in this comparative study. Five patients were excluded due to different causes: one elective case converted to radical nephrectomy due to intraoperative positive margins in the frozen section; a case of planned NSS with adrenalectomy through a thoracolumbar approach; one case with massive abdominal and pelvic lymphadenopathy; one case with liver and pancreatic resection; and one with peritoneal resection.

The preoperative imaging included renal sonography, chest x-ray, and abdominal contrast enhanced computed tomographic scan. In cases not suitable for contrast medium application, magnetic resonance imaging studies were performed. In addition, pre- and postoperative blood sample analyses, including serum levels of creatinine and hemoglobin, were performed. Patient demographics 
(gender, age), clinical characteristics (body mass index, American Society of Anesthesiologists score, tumor multifocality and PADUA score) were recorded.

The technique of retroperitoneal open NSS was as follows: Position was a full-flank position and an extended lumbar incision was made above the 11th or 12th rib; $250 \mathrm{ml}$ of $20 \%$ mannitol was applied intravenously for renal protection. Renal arteries were clamped using bulldog clamps, and regional hypothermia was established by use of crushed ice. Incision of the renal capsule was made sharply; the lesion was separated bluntly from surrounding parenchyma. A hemostatic agent was placed on the cut surface, and Vicryl sutures were used to approximate the remaining renal parenchyma with a mattress suture. Laparoscopic NSS was predominantly performed by a transperitoneal approach, only in five cases by a retroperitoneal approach. Three ports were placed when the lesion was on the left side and an additional one if it was on the right. Mannitol was applied intravenously for renal protection and renal arteries were clamped with an endo-bulldog clamp. For excision of the tumor, a choledochotome (Karl Storz, Tuttlingen, Germany) and scissors were used in warm ischemia time. Hemostasis was achieved by non-absorbable clips, central suturing and application of fibrin glue. The tumor was then removed in an endobag.

Surgical characteristics (total operative time, ischemia time, intraoperative complication, conversion of laparoscopic to open surgery and from partial to radical nephrectomy), pathological characteristics and postsurgical outcomes [difference in hemoglobin level, difference in serum creatinine level, hospital stay, and complications up to 30 days after surgery according to the modified Clavien classification (15)] were compared for both techniques. Continuous variables are given as the mean $\pm \mathrm{SD}$, categorical variables as relative frequencies. Statistical analysis was performed using Student's $t$-test for continuous variables, and Pearson chisquare test and Fisher's exact test were used to compare categorical variables, with $p \leq 0.05$ considered statistically significant. All statistics were performed using IBM SPSS Statistics v.19 (IBM Corp., Armonk, NY, USA).

\section{Results}

A total of 356 cases treated with NSS were divided into two groups, 186 laparoscopic and 170 open procedures. Both groups were compared regarding the preoperative patient data, tumor characteristics, perioperative and postoperative outcome (Table I). Overall patient characteristics did not significantly differ between LPN and OPN groups. Use of OPN was associated with higher surgical complexity, reflected by higher mean tumor diameter $(p<0.01)$, mean preoperative serum creatinine level $(p<0.01)$, solitary kidney $(p<0.01)$, multifocal tumor $(p=0.03)$ and higher PADUA score $(p<0.01)$. In accordance with increased surgical difficulty, impaired perioperative outcome was confirmed in the overall cohort. OPN was associated with prolonged operative time $(p<0.01)$, ischemia time $(p=0.02)$, and bleeding $(p=0.02)$.

Overall, 99 perioperative complications occurred in 91 patients, resulting in a complication rate of $4.3 \%$ in LPN and $8.2 \%$ in OPN, respectively $(p=0.95)$. In contrast, postoperative complication rates were $18.8 \%$ and $32.9 \%$,
Table I. Overall comparison of laparoscopic and open partial nephrectomy.

\begin{tabular}{lccc}
\hline & Laparoscopic & Open & $p$-Value \\
\hline Preoperative parameters & & & \\
Number of patients & 186 & 170 & \\
Mean age (years) & 62.3 & 61.3 & 0.49 \\
Sex (male: female) (n) & $1.8: 1$ & $1.7: 1$ & 0.76 \\
Mean BMI (kg/m $\left.{ }^{2}\right)$ & 27.5 & 27.5 & 0.92 \\
ASA score >2 (\%) & $15.6 \%$ & $20.6 \%$ & 0.22 \\
Laterality (right:left) & $1: 1.2$ & $1.1: 1$ & 0.21 \\
Mean preoperative Hb (g/dl) & 14.2 & 14 & 0.36 \\
Mean preoperative Cr (g/dl) & 0.9 & 1 & $<0.01$ \\
Mean tumor diameter (cm) & 3.1 & 4.2 & $<0.01$ \\
Single kidney (\%) & $4.3 \%$ & $13.5 \%$ & $<0.01$ \\
Multifocality (\%) & $2.7 \%$ & $7.6 \%$ & 0.03 \\
Mean PADUA score & 6.8 & 7.7 & $<0.01$ \\
& & & \\
Operative parameters & & & \\
Mean operative time (min) & 142.1 & 160.2 & $<0.01$ \\
Mean ischemia time (min) & 21 & 24 & 0.02 \\
Mean blood loss (ml) & 244 & 370 & 0.02 \\
Intraoperative complications $(\%)$ & $4.3 \%$ & $8.2 \%$ & 0.95 \\
Conversion to RN (\%) & $2.2 \%$ & $0.6 \%$ & 0.12 \\
Conversion to open (\%) & $4.8 \%$ & - & - \\
& & & \\
Postoperative outcome & & & \\
Complications (\%) & $18.8 \%$ & $32.9 \%$ & $<0.01$ \\
Mean fall in Hb (g/dl) & 2.5 & 2.7 & 0.02 \\
Mean rise in Cr (g/dl) & $0.2 \pm S D$ & 0.3 & 0.01 \\
Hospital stay (days) & $6.5 \pm S D$ & 8.6 & $<0.01$ \\
\hline
\end{tabular}

ASA: American Society of Anesthesiologists; BMI: body mass index; $\mathrm{Hb}$ : hemoglobin; Cr: creatinine; RN: radical nephrectomy.

respectively, in favor of LPN ( $p=0.01)$. Further sub-analysis revealed 23 grade I (16 OPN vs. $7 \mathrm{LPN}, p=0.03$ ), 16 grade II (11 OPN vs. 5 LPN, $p=0.09$ ), 7 grade III-A (2 OPN vs. 5 LPN, $p=0.31$ ), 33 grade III-B (19 OPN vs. 14 LPN, $p=0.24)$, 10 grade IV-A (5 OPN vs. $5 \mathrm{LPN}, p=0.89$ ), as well as one grade IV-B $(p=0.30)$ and one grade V complication $(p=0.30)$ in OPN. Furthermore, postoperative analysis of OPN showed a significant rise in serum creatinine level $(p<0.01)$, a fall in hemoglobin level $(p=0.02)$, prolonged hospital stay $(p<0.01)$ compared with LPN.

Further stratification was based on tumor complexity assessed by PADUA score $>8$ and patients with score $\leq 8$. In patients with greater tumor complexity (PADUA $>8, n=85)$, there was no significant difference between the OPN and LPN groups regarding operative and postoperative outcomes (mean operative time, mean ischemia time, mean blood loss, intraoperative complications, conversion to open surgery, mean rise in serum creatinine level, mean fall in hemoglobin level, postoperative complications and mean hospital stay) (Table II). 
Table II. Laparoscopic versus open partial nephrectomy in patients with complex tumors $(P A D U A>8)$.

\begin{tabular}{lccc}
\hline & Laparoscopic & Open & $p$-Value \\
\hline Preoperative parameters & & & \\
Number of patients & 25 & 60 & \\
Mean age (years) & 60 & 60.1 & 0.77 \\
Sex (male: female) (n) & $1.8: 1$ & $1.6: 1$ & 0.84 \\
Mean BMI (kg/m ${ }^{2}$ ) & 26.7 & 27.8 & 0.25 \\
ASA score $>$ 2 (\%) & $12 \%$ & $23.3 \%$ & 0.37 \\
Laterality (right:left) & $1: 1.1$ & $1.1: 1$ & 0.76 \\
Mean preoperative Hb (g/dl) & 14.5 & 13.8 & 0.06 \\
Mean preoperative Cr (g/dl) & 0.9 & 1 & 0.01 \\
Mean tumor diameter (cm) & 4.3 & 4.8 & 0.29 \\
Single kidney (\%) & $4 \%$ & $11.7 \%$ & 0.43 \\
Multifocality (\%) & $0 \%$ & $3.3 \%$ & 0.36 \\
& & & \\
Operative parameters & & & \\
Mean operative time (min) & 167 & 168.6 & 0.89 \\
Mean ischemia time (min) & 27.8 & 25.8 & 0.47 \\
Mean blood loss (ml) & 331 & 388 & 0.49 \\
Intraoperative complications (\%) & $16 \%$ & $5 \%$ & 0.19 \\
Conversion to RN (\%) & $12 \%$ & $1.6 \%$ & 0.07 \\
Conversion to open (\%) & $8 \%$ & 0 & - \\
Postoperative outcome & & & \\
Complications (\%) & & & \\
Mean fall in Hb (g/dl) & $28 \%$ & $38.3 \%$ & 0.36 \\
Mean rise in Cr (g/dl) & 2.7 & 2.9 & 0.56 \\
Hospital stay (days) & 0.3 & 0.4 & 0.52 \\
\hline & 8.3 & 9.8 & 0.20 \\
\hline
\end{tabular}

ASA: American Society of Anesthesiologists; BMI: body mass index; $\mathrm{Hb}$ : hemoglobin; Cr: creatinine; RN: radical nephrectomy.

Interestingly, superiority of LPN was confirmed after stratification for imperative indication (single kidney/ preoperative serum creatinine $>1.2 \mathrm{mg} / \mathrm{dl} \mathrm{n}=71$ ) or presence of tumor multifocality. In this subgroup, LPN was superior to conventional surgery due to its significant improvements in operative time $(p=0.01)$, blood loss $(p=0.02)$, hemoglobin level $(p=0.04)$, postoperative complications $(p=0.03)$, and mean hospital stay $(p=0.03)$ (Table III).

\section{Discussion}

The open approach is the standard technique for NSS. Many studies reported that laparoscopic NSS has a longer renal ischemia time, and higher intra- and postoperative complication rates when compared to open NSS $(9,16)$. However, contradictory data for laparoscopic NSS exist indicating a shorter operative time, a reduced operative blood loss and a shorter hospital stay (12). An obvious decrease in the overall complication rate of LPN (33\% in earlier vs. $19 \%$ in the present series) has occurred in the past few years (17). Subsequently, recent studies have demonstrated that there is
Table III. Comparison of laparoscopic versus open partial nephrectomy in patients with imperative indication or multifocal tumor.

\begin{tabular}{lccc}
\hline & Laparoscopic & Open & $p$-Value \\
\hline Preoperative parameters & & & \\
Number of patients & 24 & 47 & \\
Mean age (years) & 67.04 & 64.55 & 0.40 \\
Sex (male: female) (n) & $3: 1$ & $3.7: 1$ & 0.72 \\
Mean BMI (kg/m $\left.{ }^{2}\right)$ & 27.1 & 28.7 & 0.16 \\
ASA score $>$ 2 (\%) & 29.2 & 23.4 & 0.60 \\
Laterality (right:left) & $1: 1.2$ & $1: 1$ & 0.68 \\
Mean preoperative Hb (g/dl) & 13.7 & 13.9 & 0.59 \\
Mean preoperative Cr (g/dl) & 1.3 & 1.4 & 0.25 \\
Mean tumor diameter (cm) & 2.7 & 3.6 & 0.08 \\
Single kidney (\%) & 33.3 & 48.9 & 0.21 \\
Multifocality (\%) & 16.7 & 27.6 & 0.39 \\
& & & \\
Operative parameters & & & \\
Mean operative time (min) & 133.5 & 166.7 & 0.01 \\
Mean ischemia time (min) & 17 & 16.3 & 0.85 \\
Mean blood loss (ml) & 117 & 495 & 0.02 \\
Intraoperative complications (\%) & $0 \%-$ & $4.3 \%$ & 0.31 \\
Conversion to RN (\%) & $0 \%$ & $0 \%$ & - \\
Conversion to open (\%) & $8.3 \%$ & $0 \%$ & - \\
Postoperative outcome & & & \\
Complications (\%) & & & \\
Mean fall in Hb (g/dl) & $16.7 \%$ & $44.7 \%$ & 0.03 \\
Mean rise in Cr (g/dl) & 2.1 & 2.7 & 0.04 \\
Hospital stay (days) & 0.2 & 0.4 & 0.08 \\
\hline & 6.1 & 9.2 & $<0.01$ \\
\hline
\end{tabular}

ASA: American Society of Anesthesiologists; BMI: body mass index; Hb: hemoglobin; Cr: creatinine; RN: radical nephrectomy.

no detectable difference between open and laparoscopic NSS regarding the perioperative outcomes $(18,19)$. In addition, shorter ischemia time and a lower complication rate was reported for laparoscopic NSS $(7,14)$.

However, these results might be misleading due to lack of reporting of detailed tumor characteristics.

In the present analysis of consecutive recruited patients LPN was more frequently used in patients with great tumor complexity. Although the characteristics of the patients themselves were well balanced between the two groups, more complex tumor characteristics reflected by higher PADUA scores, a higher percentage of those with a solitary kidney and multifocal tumor lesions, with raised preoperative creatinine level, were observed in the OPN cohort. Considering this preoperative patient selection in accordance with recommended standards (20), as expected, LPN was associated with a significantly shorter duration of operative and ischemia time, as well as hospital stay, and a lower blood loss as determined by fall in hemoglobin level in overall analysis. Furthermore, the rate of intraoperative and postoperative complications was significantly lower when 
compared to the open surgery group. A further subgroup analysis was carried out to exclude the aforementioned selection bias. We concentrated our sub-analysis on patients with highest surgical complexity based on anatomical tumor (PADUA >8) and individual risk factors (imperative indication and/or multifocal tumor growth) based on our previous research (21). Interestingly, LPN retained its superiority in this patient group regarding operative time, blood loss, fall in hemoglobin level, postoperative complication rate and hospital stay. These benefits were already postulated beyond established indications for elective NSS (22). However, there is only minor evidence in patients with imperative surgery due to patient selection criteria (23).

Although our results suggest a potential perioperative superiority of LPN, especially in patients with imperative indication (single kidney or preoperative serum creatinine $>1.2 \mathrm{mg} / \mathrm{dl}$ ), long-term data regarding renal function and oncological safety are lacking. Nevertheless, a possible advantage for long-term renal function might be at least partially expected by the decreased vulnerability from LPN by reducing blood loss, operative and ischemia time. The observed superiority of LPN might be at least partly explained by these facts. In contrast, for patients with elective indication and a PADUA score $>8$, there was no significant difference between the open and laparoscopic approaches. In line with this, a recent study found comparable perioperative and functional outcomes for minimally-invasive and open NSS in patients with moderate and highly complex lesions (24). Furthermore, different minimally-invasive surgical modalities did not differ in surgical and pathological outcomes between robot-assisted and LPN in patients with moderate to complex renal tumors in a prospective trial (25).

Although our study had inherent limitations, including its retrospective character and limited sample size, this series will hopefully strengthen the role of minimally invasive NSS. In conclusion, open surgery is still a preferred option in patients with higher tumor complexity, while LPN was equivalent to OPN in patients with a high PADUA score. Moreover, the overall performance of LPN reflected by operative and postoperative outcomes seems be superior to that of open surgery, even in patients with imperative indication or multifocal tumor burden.

\section{References}

1 Ljungberg B, Bensalah K, Canfield S, Dabestani S, Hofmann F, Hora M, Kuczyk MA, Lam T, Marconi L, Merseburger AS, Mulders P, Powles T, Staehler M, Volpe A and Bex A: EAU guidelines on renal cell carcinoma: 2014 update. Eur Urol 67(5): 913-924, 2015.

2 MacLennan S, Imamura M, Lapitan MC, Omar MI, Lam TB, Hilvano-Cabungcal AM, Royle P, Stewart F, MacLennan G, MacLennan SJ, Dahm P, Canfield SE, McClinton S, Griffiths TR, Ljungberg B and N'Dow J: Systematic review of perioperative and quality-of-life outcomes following surgical management of localised renal cancer. Eur Urol 62(6): 1097$1117,2012$.

3 Becker F, Roos FC, Janssen M, Brenner W, Hampel C, Siemer S, Thuroff JW and Stockle M: Short-term functional and oncologic outcomes of nephron-sparing surgery for renal tumours $\geq 7 \mathrm{~cm}$. Eur Urol 59(6): 931-937, 2011.

4 Simmons MN, Weight CJ and Gill IS: Laparoscopic radical versus partial nephrectomy for tumors $>4 \mathrm{~cm}$ : Intermediate-term oncologic and functional outcomes. Urology 73(5): 1077-1082, 2009.

5 Margulis V, Tamboli P, Jacobsohn KM, Swanson DA and Wood CG: Oncological efficacy and safety of nephron-sparing surgery for selected patients with locally advanced renal cell carcinoma. BJU Int 100(6): 1235-1239, 2007.

6 Aron M and Gill IS: Minimally invasive nephron-sparing surgery (MINSS) for renal tumours part i: Laparoscopic partial nephrectomy. Eur Urol 51(2): 337-346; discussion 346-337, 2007.

7 Marszalek M, Meixl H, Polajnar M, Rauchenwald M, Jeschke K and Madersbacher S: Laparoscopic and open partial nephrectomy: A matched-pair comparison of 200 patients. Eur Urol 55(5): 1171-1178, 2009.

8 Schwentner C AS, Radmayr C, Colleselli D, Schilling D, Sievert $\mathrm{KD}$ and Stenzl A: Contemporary minimally invasive nephron sparing surgery. Central European Journal of Urology 62(4): 222-227, 2009.

9 Gill IS, Matin SF, Desai MM, Kaouk JH, Steinberg A, Mascha E, Thornton J, Sherief MH, Strzempkowski B and Novick AC: Comparative analysis of laparoscopic versus open partial nephrectomy for renal tumors in 200 patients. J Urol 170(1): 6468, 2003.

10 Becker A, Pradel L, Kluth L, Schmid M, Eichelberg C, Ahyai S, Trinh Q, Seiler D, Dahlem R, Hansen J, Rink M, Zacharias M, Mehnert A, Bergelt C, Fisch M and Chun FK: Laparoscopic versus open partial nephrectomy for clinical T1 renal masses: No impact of surgical approach on perioperative complications and long-term postoperative quality of life. World J Urol 33(3): 421-426, 2015.

11 Feder MT, Patel MB, Melman A, Ghavamian R and Hoenig DM: Comparison of open and laparoscopic nephrectomy in obese and non-obese patients: Outcomes stratified by body mass index. J Urol 180(1): 79-83, 2008.

12 Gill IS, Kavoussi LR, Lane BR, Blute ML, Babineau D, Colombo JR, Jr., Frank I, Permpongkosol S, Weight CJ, Kaouk JH, Kattan MW and Novick AC: Comparison of 1,800 laparoscopic and open partial nephrectomies for single renal tumors. J Urol 178(1): 41-46, 2007.

13 Lane BR, Novick AC, Babineau D, Fergany AF, Kaouk JH and Gill IS: Comparison of laparoscopic and open partial nephrectomy for tumor in a solitary kidney. J Urol 179(3): 847851; discussion 852, 2008.

14 Sun M, Abdollah F, Shariat SF, Schmitges J, Trinh QD, Tian Z, Jeldres C, Abdo A, Bianchi M, Briganti A, Montorsi F, Perrotte $\mathrm{P}$ and Karakiewicz PI: Propensity-score matched comparison of complications, blood transfusions, length of stay, and in-hospital mortality between open and laparoscopic partial nephrectomy: A national series. Eur J Surg Oncol 38(1): 80-87, 2012.

15 Dindo D, Demartines N and Clavien P-A: Classification of surgical complications: A new proposal with evaluation in a cohort of 6336 patients and results of a survey. Ann Surg 240(2): 205-213, 2004 
16 Porpiglia F, Volpe A, Billia M and Scarpa RM: Laparoscopic versus open partial nephrectomy: Analysis of the current literature. Eur Urol 53(4): 732-742; discussion 742-733, 2008.

17 Simmons MN and Gill IS: Decreased complications of contemporary laparoscopic partial nephrectomy: Use of a standardized reporting system. J Urol 177(6): 2067-2073; discussion 2073, 2007.

18 Breda A, Finelli A, Janetschek G, Porpiglia F and Montorsi F: Complications of laparoscopic surgery for renal masses: Prevention, management, and comparison with the open experience. Eur Urol 55(4): 836-850, 2009.

19 Sprenkle PC, Power N, Ghoneim T, Touijer KA, Dalbagni G, Russo P and Coleman JA: Comparison of open and minimally invasive partial nephrectomy for renal tumors 4-7 centimeters. Eur Urol 61(3): 593-599, 2012.

20 Heuer R, Gill IS, Guazzoni G, Kirkali Z, Marberger M, Richie JP and de la Rosette JJ: A critical analysis of the actual role of minimally invasive surgery and active surveillance for kidney cancer. Eur Urol 57(2): 223-232, 2010.

21 Kruck S, Anastasiadis AG, Walcher U, Stenzl A, Herrmann TR and Nagele U: Laparoscopic partial nephrectomy: Risk stratification according to patient and tumor characteristics. World J Urol 30(5): 639-646, 2012.

22 Roos FC, Brenner W, Thomas C, Jager W, Thuroff JW, Hampel $\mathrm{C}$ and Jones J: Functional analysis of elective nephron-sparing surgery $v s$. radical nephrectomy for renal tumors larger than 4 cm. Urology 79(3): 607-613, 2012.
23 Pignot G, Bigot P, Bernhard JC, Bouliere F, Bessede T, Bensalah K, Salomon L, Mottet N, Bellec L, Soulie M, Ferriere JM, Pfister C, Drai J, Colombel M, Villers A, Rigaud J, Bouchot O, Montorsi F, Bertini R, Belldegrun AS, Pantuck AJ and Patard JJ: Nephron-sparing surgery is superior to radical nephrectomy in preserving renal function benefit even when expanding indications beyond the traditional 4-cm cutoff. Urol Oncol 32(7): 1024-1030, 2014.

24 Gupta GN, Boris R, Chung P, Linehan WM, Pinto PA and Bratslavsky G: Robot-assisted laparoscopic partial nephrectomy for tumors greater than $4 \mathrm{~cm}$ and high nephrometry score: Feasibility, renal functional, and oncological outcomes with minimum 1-year follow-up. Urol Oncol 31(1): 51-56, 2013.

25 Masson-Lecomte A, Bensalah K, Seringe E, Vaessen C, de la Taille A, Doumerc N, Rischmann P, Bruyere F, Soustelle L, Droupy $\mathrm{S}$ and Roupret M: A prospective comparison of surgical and pathological outcomes obtained after robot-assisted or pure laparoscopic partial nephrectomy in moderate to complex renal tumours: Results from a French multicentre collaborative study. BJU Int 111(2): 256-263, 2013
Received November 8, 2016

Revised November 27, 2016

Accepted November 29, 2016 